\title{
Response of the Hadley Circulation to Climate Change in an Aquaplanet GCM Coupled to a Simple Representation of Ocean Heat Transport
}

\author{
XAVIER J. LEVINE AND TAPIO SCHNEIDER \\ California Institute of Technology, Pasadena, California
}

(Manuscript received 18 May 2010, in final form 5 November 2010)

\begin{abstract}
It is unclear how the width and strength of the Hadley circulation are controlled and how they respond to climate changes. Simulations of global warming scenarios with comprehensive climate models suggest the Hadley circulation may widen and weaken as the climate warms. But these changes are not quantitatively consistent among models, and how they come about is not understood. Here, a wide range of climates is simulated with an idealized moist general circulation model (GCM) coupled to a simple representation of ocean heat transport, in order to place past and possible future changes in the Hadley circulation into a broader context and to investigate the mechanisms responsible for them.

By comparison of simulations with and without ocean heat transport, it is shown that it is essential to take low-latitude ocean heat transport and its coupling to wind stress into account to obtain Hadley circulations in a dynamical regime resembling Earth's, particularly in climates resembling present-day Earth's and colder. As the optical thickness of an idealized longwave absorber in the simulations is increased and the climate warms, the Hadley circulation strengthens in colder climates and weakens in warmer climates; it has maximum strength in a climate close to present-day Earth's. In climates resembling present-day Earth's and colder, the Hadley circulation strength is largely controlled by the divergence of angular momentum fluxes associated with eddies of midlatitude origin; the latter scale with the mean available potential energy in midlatitudes. The importance of these eddy momentum fluxes for the Hadley circulation strength gradually diminishes as the climate warms. The Hadley circulation generally widens as the climate warms, but at a modest rate that depends sensitively on how it is determined.
\end{abstract}

\section{Introduction}

The Hadley circulation appears to have widened in the past decades (Hu and Fu 2007; Seidel and Randel 2007; Seidel et al. 2008), and it also widens in many global warming simulations (Lu et al. 2007; Seager et al. 2007). It may also have strengthened, though the evidence for a significant strengthening is ambiguous (Quan et al. 2004; Chen et al. 2002; Mitas and Clement 2005). In global warming simulations, the Hadley circulation weakens slightly in many models, but this is not consistent among all models (Tanaka et al. 2005). Trends in the Hadley circulation inferred from observational data are considerably larger than those obtained in comprehensive general circulation model (GCM) simulations (Mitas and Clement 2005; Johanson and Fu 2009). Depending on how it is

Corresponding author address: Xavier Levine, California Institute of Technology, 1200 E. California Blvd., Pasadena, CA 91125. E-mail: xavier@caltech.edu measured, the widening appears to be nearly an order of magnitude larger in observations than in GCM simulations (Seidel et al. 2008). Yet it is unclear what instrumental or modeling artifact causes such large discrepancies in the response of the Hadley circulation to climate change; radiosonde data and their use in reanalysis models may be part of the problem (see, e.g., Santer et al. 2005; Sherwood 2007).

Despite a large body of observations and numerous studies with GCMs, it remains unclear how the width and strength of the Hadley circulation are controlled (Schneider 2006). Here, we study how the width and strength of the Hadley circulation change over a wide range of climates simulated with an idealized GCM. This allows us to place possible changes in the Hadley circulation in a larger context and to determine whether, for example, the Hadley circulation generally widens as the climate warms, even in climates very different from the present. Our intention with these simulations is to provide a detailed target for the development of a Hadley circulation theory, but such a theory itself is beyond the scope of this paper. 
Equinox mean

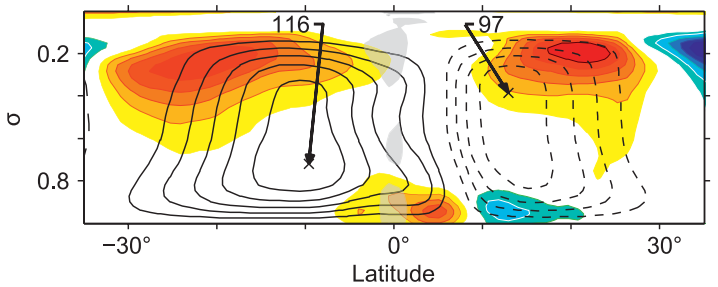

Annual mean

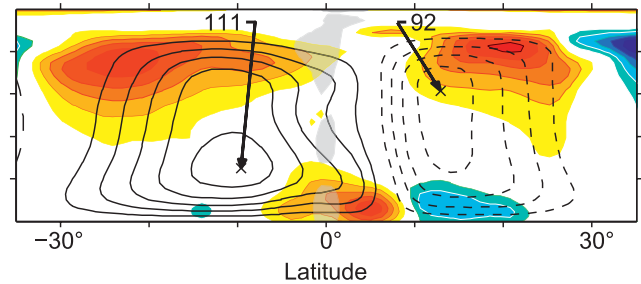

FIG. 1. Earth's Hadley circulation (a) averaged over equinox seasons [March-May (MAM) and SeptemberNovember (SON)] and (b) averaged annually. Black contours show the mass flux streamfunction, with dashed (negative) contours indicating clockwise motion and solid (positive) contours indicating counterclockwise motion. The contour interval is $25 \mathrm{~Sv}=25 \times 10^{9} \mathrm{~kg} \mathrm{~s}^{-1}$. Arrows indicate the streamfunction extremum in each hemisphere, with the magnitude given in $10^{9} \mathrm{~kg} \mathrm{~s}^{-1}$. Colors indicate horizontal eddy momentum flux divergence $\operatorname{div}\left(\overline{u^{\prime} v^{\prime}} \cos \phi\right)$, with the overbar denoting a temporal and zonal mean and primes denoting deviations therefrom (i.e., eddy fields include stationary and transient eddies). The contour interval for the eddy momentum flux divergence is $4 \times 10^{-6} \mathrm{~m} \mathrm{~s}^{-2}$, with red tones for positive and blue tones for negative values; the darkest red tone corresponds to values greater than $2.4 \times 10^{-5} \mathrm{~m} \mathrm{~s}^{-2}$. Gray shading indicates regions where $|\mathrm{Ro}|>0.5$. Flow statistics are computed from reanalysis data for the years 1980-2001 provided by the European Centre for Medium-Range Weather Forecasts (Kallberg et al. 2004; Uppala et al. 2005).

It is often assumed that the Hadley circulation responds directly to changes in thermal driving, in the sense that its changes are controlled by thermodynamic balances. For that to be the case, the balance of angular momentum about Earth's spin axis, or the balance of zonal momentum, would have to be degenerate in the upper troposphere, so that eddy momentum fluxes do not influence the Hadley circulation. In the upper troposphere, above the center of the Hadley cells where vertical momentum advection by the mean meridional circulation can be neglected, the mean zonal momentum balance may be used to quantify how strongly eddy momentum fluxes influence the mean meridional flow:

$$
f(1-\mathrm{Ro}) \bar{v} \approx S_{e} .
$$

Here, $S_{e}$ is the eddy momentum flux divergence, $f$ the planetary vorticity (Coriolis parameter), and $v$ the meridional velocity; overbars indicate a temporal and zonal mean. The local Rossby number Ro $=-\bar{\zeta} / f$, with relative vorticity $\zeta$, is a nondimensional measure of the importance of nonlinear angular momentum advection by the mean meridional circulation (Walker and Schneider 2006; Schneider 2006). In the limit Ro $\rightarrow 1$, the upper branch of the Hadley circulation conserves angular momentum and is unaffected by eddy momentum fluxes; its strength responds directly to changes in thermal driving. This is the limit considered in classical theories for the Hadley circulation, which provide expressions for its width and strength as a function of thermal and other parameters (Schneider 1977; Held and Hou 1980; Lindzen and Hou 1988). In the limit Ro $\rightarrow 0$, the strength of the Hadley circulation is controlled by eddy momentum fluxes; its strength responds to changes in thermal driving only insofar as they affect the eddy momentum fluxes (e.g., Dickinson 1971). In between these limiting cases lie Hadley circulations with $0<$ Ro $<1$ in their upper branches, which respond to changes in thermal driving through changes in thermodynamic balances, in eddy momentum fluxes, and possibly in Rossby numbers. There is no theory that captures how the Hadley circulation responds to climate changes in this intermediate range of Rossby numbers.

Figure 1 shows the meridional mass flux streamfunction, the eddy momentum flux divergence, and the local Rossby number for Earth's atmosphere during equinox and in the annual mean. The quantities are similar during equinox and in the annual mean, suggesting that the annual mean is not dominated by an average of solstitial circulations (cf. Lindzen and Hou 1988; see also Dima and Wallace 2003; Walker and Schneider 2005). The eddy momentum flux divergence has a broad maximum in the upper troposphere centered near $21^{\circ}$ latitude, extending deep into the Hadley cells (color contours in Fig. 1). The Rossby number in the upper troposphere gradually decreases from close to 1 at the equator to close to 0 in the subtropics, with values greater than 0.5 confined to a narrow band within $\sim 4^{\circ}$ latitude of the equator (gray shading in Fig. 1). Near the center of the Hadley cells (near $10^{\circ}$ latitude), the Rossby number is between 0.35 and 0.45 during equinox and in the annual mean. This indicates that angular momentum-conserving theories are inadequate for describing Hadley cell dynamics, at least poleward of about $10^{\circ}$ latitude during equinox and in the annual mean. Understanding how eddy momentum fluxes change with climate therefore is integral to understanding how the Hadley circulation responds to climate changes. This is borne out in observational data and models, which show that interannual variations in the strength of Hadley cells and 
differences in their strength among climate models are correlated with the eddy momentum flux divergence in low latitudes (Caballero 2007, 2008).

In addition to eddy momentum fluxes, the Hadley circulation is intimately linked to ocean heat transport, which dominates the total meridional energy transport in the ocean-atmosphere system at low latitudes (Trenberth and Caron 2001). The ocean heat transport is coupled to the strength of the Hadley circulation: The Coriolis force on the meridional near-surface mass flux in the Hadley cells approximately balances the zonal surface wind stress (Ekman balance in the atmosphere). In turn, the zonal surface wind stress, by Ekman balance in the ocean, induces an ocean circulation with upwelling in the deep tropics, poleward flow near the surface, downwelling in the subtropics, and an equatorward return flow in the thermocline (McCreary and Lu 1994; Lu et al. 1998). This meridional overturning cell dominates the low-latitude ocean heat transport and helps control the surface temperature near the equator (Klinger and Marotzke 2000; Held 2000) and the meridional surface temperature gradients (Trenberth and Solomon 1994). It also dominates the total heat transport in the deep tropics because the Hadley circulation there is inefficient at transporting energy (e.g., Peixoto and Oort 1992, chapter 13). Taking this heat transport into account is essential for obtaining Hadley circulations resembling Earth's. If it is neglected in simulations, meridional surface temperature gradients near the equator are generally too large, resulting in Hadley circulations that are too strong and often are closer to the angular momentum-conserving limit Ro $\rightarrow 1$ than is Earth's Hadley circulation. This occurs, for example, in the simulations in Herweijer et al. (2005) and Lee et al. (2008), potentially implying different responses to climate changes depending on whether ocean heat transport is taken into account or not.

We investigate how the Hadley circulation responds to climate changes with an idealized GCM coupled to a simple representation of ocean heat transport by a winddriven, low-latitude overturning circulation. We consider the case of insolation with no diurnal or seasonal cycle-the natural starting point for studies of the Hadley circulation, before considering the additional complexities of the seasonal cycle (e.g., Lindzen and Hou 1988; Walker and Schneider 2005; Schneider and Bordoni 2008; Bordoni and Schneider 2008). Inclusion of ocean heat transport allows us to obtain Hadley circulations with strengths, Rossby numbers, and widths resembling those of Earth, and coupling it to the surface wind stress and thus to the strength of the Hadley circulation allows us to take into account feedbacks between the atmosphere and low-latitude oceans. Using this GCM, we simulate a wide range of climates by varying the longwave optical thickness of the atmosphere. We compare these simulations with simulations performed by O'Gorman and Schneider (2008b) that are otherwise identical but do not include ocean heat transport. We show that the Hadley circulation generally widens as the climate warms but that it does not necessarily weaken, both in the simulations with ocean transport and in those of O'Gorman and Schneider without ocean heat transport. We analyze the dynamics involved in the changes in some detail to provide a target for the development of theories to account for them. (O'Gorman and Schneider focused on the hydrologic cycle and did not analyze Hadley circulation dynamics in detail. Here, we analyze their simulations along with the ones without ocean heat transport.)

\section{Idealized GCM}

\section{a. Atmosphere}

The atmospheric GCM is a primitive equation model with idealized representations of radiative transfer (a gray radiation scheme) and moist thermodynamics (only the vapor-liquid phase transition of water is taken into account). It is based on the Flexible Modeling System (FMS) developed at the Geophysical Fluid Dynamics Laboratory (GFDL). The model is described in detail in O'Gorman and Schneider (2008b); it is similar to the model described in Frierson et al. (2006) and Frierson (2007). Here, we only give a brief overview.

The GCM solves the primitive equations in vorticitydivergence form, using the spectral-transform method in the horizontal (Bourke 1974) and finite differences in the vertical (Simmons and Burridge 1981). We use a horizontal resolution of T42 and 30 vertical sigma levels, unevenly spaced to better resolve dynamics in the uppermost and lowermost part of the troposphere. To damp small scales, scale-selective $\nabla^{8}$ hyperdiffusion is included in the vorticity, divergence, and temperature equations, with a hyperdiffusion coefficient chosen such that the damping time scale for the smallest resolved scale is $12 \mathrm{~h}$.

The lower boundary of the GCM is uniform and water covered, with an albedo of 0.38 . A planetary boundary layer scheme with Monin-Obhukov surface fluxes, which depend on the stability of the boundary layer, links atmospheric dynamics to surface fluxes of momentum, latent heat, and sensible heat. The roughness length for momentum fluxes is $5 \times 10^{-3} \mathrm{~m}$ but that for latent and sensible heat fluxes is reduced to $10^{-5} \mathrm{~m}$ to obtain energy fluxes and a climate similar to Earth's in the aquaplanet setting of our simulations.

Radiative transfer is represented by a two-stream gray radiation scheme with longwave and shortwave optical 
depths that only depend on latitude and pressure. The longwave absorption in this scheme can be thought of as an idealized representation of the longwave absorption by water vapor and well-mixed greenhouse gases. However, the longwave optical thickness of the atmosphere is a fixed function of latitude, which we rescale to change the simulated climate but which does not depend, for example, on the atmospheric concentration of water vapor in the model. Thus, we do not take water vapor feedback explicitly into account, but its effect can be viewed as included in the prescribed longwave optical thickness for a given climate. Moist convection is represented by a slightly modified version of the quasiequilibrium convection scheme of Frierson (2007). It relaxes, with a time scale of $2 \mathrm{~h}$, grid-scale temperature profiles toward a moist-adiabatic profile and water vapor profiles toward a profile with fixed relative humidity $(70 \%)$, whenever a parcel lifted from the lowest model level is convectively unstable. Large-scale condensation removes water vapor from the atmosphere when the specific humidity on the grid scale exceeds the saturation specific humidity.

In addition to neglecting surface inhomogeneities such as land-ocean contrasts and topography, the GCM is idealized in that it neglects explicit representations of features such as sea ice, aerosols, and clouds (except insofar as their global-mean effect on Earth's albedo is implicitly taken into account in our choice of surface albedo).

\section{b. Ocean}

The surface wind stress from the atmosphere model drives a simple model of ocean heat transport in low latitudes. The ocean heat transport in the equatorial latitude band with surface easterlies is assumed to be accomplished by a wind-driven meridional overturning circulation, with poleward flow near the surface and an equatorward return flow in the thermocline. The Coriolis force on the poleward surface flow is in balance with the zonal surface wind stress (Ekman balance). The return flow is assumed to be adiabatic, so that water parcels conserve their temperature (neglecting salinity and compressibility effects) after subduction in the subtropics, until they upwell into the surface mixed layer in the deep tropics. Klinger and Marotzke (2000) showed that under these conditions, the meridional heat flux associated with the wind-driven ocean circulation can be expressed in terms of surface quantities (subscript $s$ ) as

$$
\begin{aligned}
F(\varphi) & =2 \pi a c_{o} \cos \varphi \int_{-H}^{0} \rho_{o} \bar{v} \bar{T} d z \\
& =2 \pi a c_{o} \cos \varphi \int_{\bar{T}_{s}\left(\varphi_{h}\right)}^{\bar{T}_{s}(\varphi)} \frac{\tau\left(\bar{T}_{s}\right)}{f} d \bar{T}_{s} .
\end{aligned}
$$

Here, $F(\varphi)$ is the meridional heat flux through a latitude $(\varphi)$ circle, integrated from the surface to the ocean depth $H, v$ is the meridional wind, $T$ is the temperature, $c_{o}$ is the specific heat, $\rho_{o}$ is the density of the ocean, $\tau$ is the zonal surface wind stress, $\varphi_{h}$ is the closest latitude to the equator at which zonal surface winds change sign (from easterlies to westerlies), and $a$ is Earth's radius. Implicit in this formula is that the mean temperature of the subsurface return flow at $\varphi$ is expressed as the average surface temperature poleward of $\varphi$, with the average weighted by the strength of the downwelling Ekman mass flux at the poleward latitudes (Klinger and Marotzke 2000; Held 2000). This simple model of low-latitude ocean heat transport assumes laminar flow (no eddy mixing), with Ekman upwelling confined to the equator.

If this heat flux is coupled directly to the atmospheric GCM through the surface temperature and wind stress, it results in a local surface temperature minimum at the equator and a displacement of the ascending branches of the Hadley circulation a few degrees off the equator. Double ITCZs occur in coupled ocean-atmosphere GCMs and might be a natural state for an atmosphere-ocean system in perpetual equinox or in the annual mean. In our model, however, this ocean heat transport would imply excessive cooling at the equator, in part caused by assuming Ekman balance at the equator although the Coriolis parameter vanishes there. As a remedy, we apply a Gaussian smoothing filter to obtain the smoothed divergence of the meridional ocean heat transport:

$$
\widetilde{\nabla \cdot F}=\int_{-\pi / 2}^{\pi / 2} \frac{1}{a \cos \varphi^{\prime}} \partial_{\varphi}\left[F\left(\varphi^{\prime}\right) \cos \varphi^{\prime}\right] P\left(\varphi, \varphi^{\prime}\right) d \varphi^{\prime} .
$$

Here,

$$
P\left(\varphi, \varphi^{\prime}\right)=\frac{1}{Z} \exp \left[\frac{-\left(\varphi^{\prime}-\varphi\right)^{2}}{2 s^{2}}\right]
$$

is a Gaussian smoothing function, with normalization factor $Z$ chosen such that

$$
\int_{-\pi / 2}^{\pi / 2} P\left(\varphi^{\prime}, \varphi\right) d \varphi^{\prime}=1 .
$$

We chose the standard deviation $s=7^{\circ}$ for the smoothing filter.

The ocean heat transport in this representation is confined to the equatorial latitude band with surface easterlies (essentially the region of the Hadley circulation), which in the GCM is determined at every time step before the heat transport is calculated. In higher latitudes, the ocean heat transport vanishes, and the ocean 

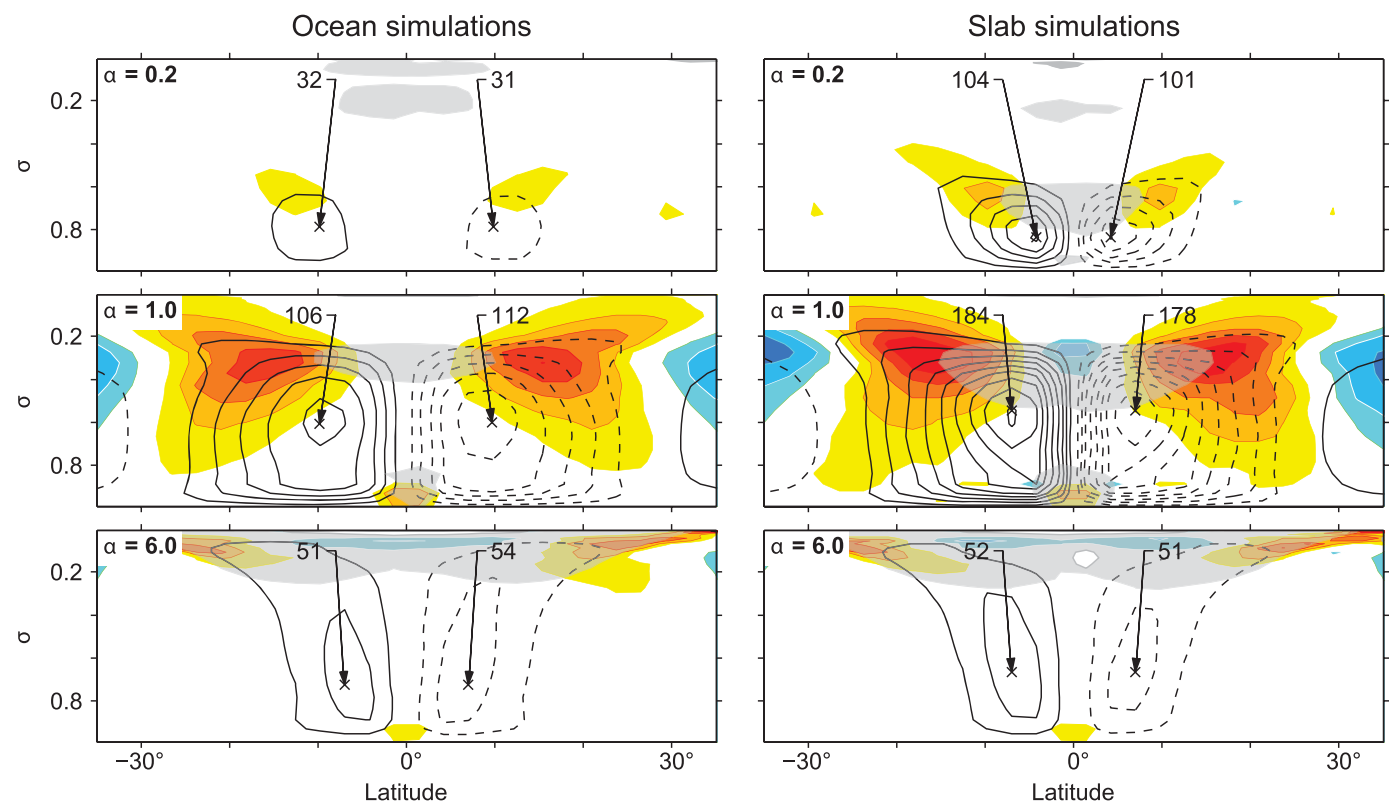

FIG. 2. Mass flux streamfunction (black contours) and horizontal eddy momentum flux divergence $\operatorname{div}\left(\overline{u^{\prime} v^{\prime}} \cos \phi\right)(\operatorname{color}$ contours). Plotting conventions and contour intervals are as in Fig. 1. Rows show (top) the coldest ( $\alpha=0.2)$, (middle) the reference $(\alpha=1.0)$, and (bottom) the warmest $(\alpha=6.0)$ simulation both (left) with and (right) without ocean heat transport. Differences between the Northern and Southern Hemisphere are indicative of sampling variability.

is treated as a static thermal slab. The thermal inertia of the ocean is that of a water slab $0.5 \mathrm{~m}$ deep.

In a real ocean without lateral boundaries, to conserve momentum a stress of equal magnitude but opposite sign as the surface stress would be required at the bottom of the ocean; this would generally mean that the meridional overturning circulation would have to close in a frictional bottom boundary layer rather than in the thermocline (Haynes et al. 1991; O'Gorman and Schneider 2008c). We will ignore this complication here and assume we can close the circulation within the thermocline with adiabatic flow.

\section{c. Simulations}

The GCM is forced by Earth's annual-mean insolation at the top of the atmosphere. This insolation is steady (i.e., there is no diurnal or seasonal cycle). Each simulation is run for more than 1000 days. Circulation statistics are averages of fields over the last 300 days, sampled 4 times daily, after a statistically steady state has been reached. Because the Northern and Southern Hemispheres in the simulations are statistically identical, hemispheric differences in the zonal- and time-mean fields shown in some of the figures are indicative of lowfrequency sampling variability.

\section{Reference climate}

To illustrate the impact of ocean heat transport on the low-latitude climate, we compare the climatology of the tropical atmosphere in reference simulations with the simple wind-coupled oceanic heat flux ("ocean simulation") and without any ocean heat transport ("slab simulation"). Figure 2 (middle row) show the mass flux streamfunction, eddy momentum flux divergence, and the Rossby number in the two reference simulations. The Hadley circulation mass flux in the ocean simulation is $109 \mathrm{~Sv}$ ( $1 \mathrm{~Sv} \equiv$ $10^{6} \mathrm{~m}^{3} \mathrm{~s}^{-1}$ ), which compares very well with that of Earth in the annual mean (102 Sv), shown in Fig. 1. The mass flux is $66 \%$ stronger in the slab simulation than in the ocean simulation.

Ocean heat transport also decreases the Rossby number in the upper branch of the Hadley circulation, especially in the deep tropics. Figure 2 shows that the region of large Rossby number is smaller in the ocean simulation than in the slab simulation. The Rossby number in the deep tropics is also smaller in the ocean simulation. At the center of the Hadley circulation, the Rossby number in the ocean simulation $(\sim 0.4)$ is closer to Earth's in the annual mean $(\sim 0.3)$ than it is in the slab simulation $(\sim 0.6)$. This reduction in Rossby number implies changes in the mean zonal wind in the upper troposphere: Fig. 3 (middle row) shows a decrease in the strength of the subtropical jet, from $36 \mathrm{~m} \mathrm{~s}^{-1}$ in the slab simulation to $29 \mathrm{~m} \mathrm{~s}^{-1}$ in the ocean simulation. The eddy momentum flux divergence is also influenced by ocean heat transport, but to a lesser extent than the maximum mass flux streamfunction or the Rossby number. Figure 2 (middle row) shows that, in the subtropics (poleward of $15^{\circ}$ latitude), 

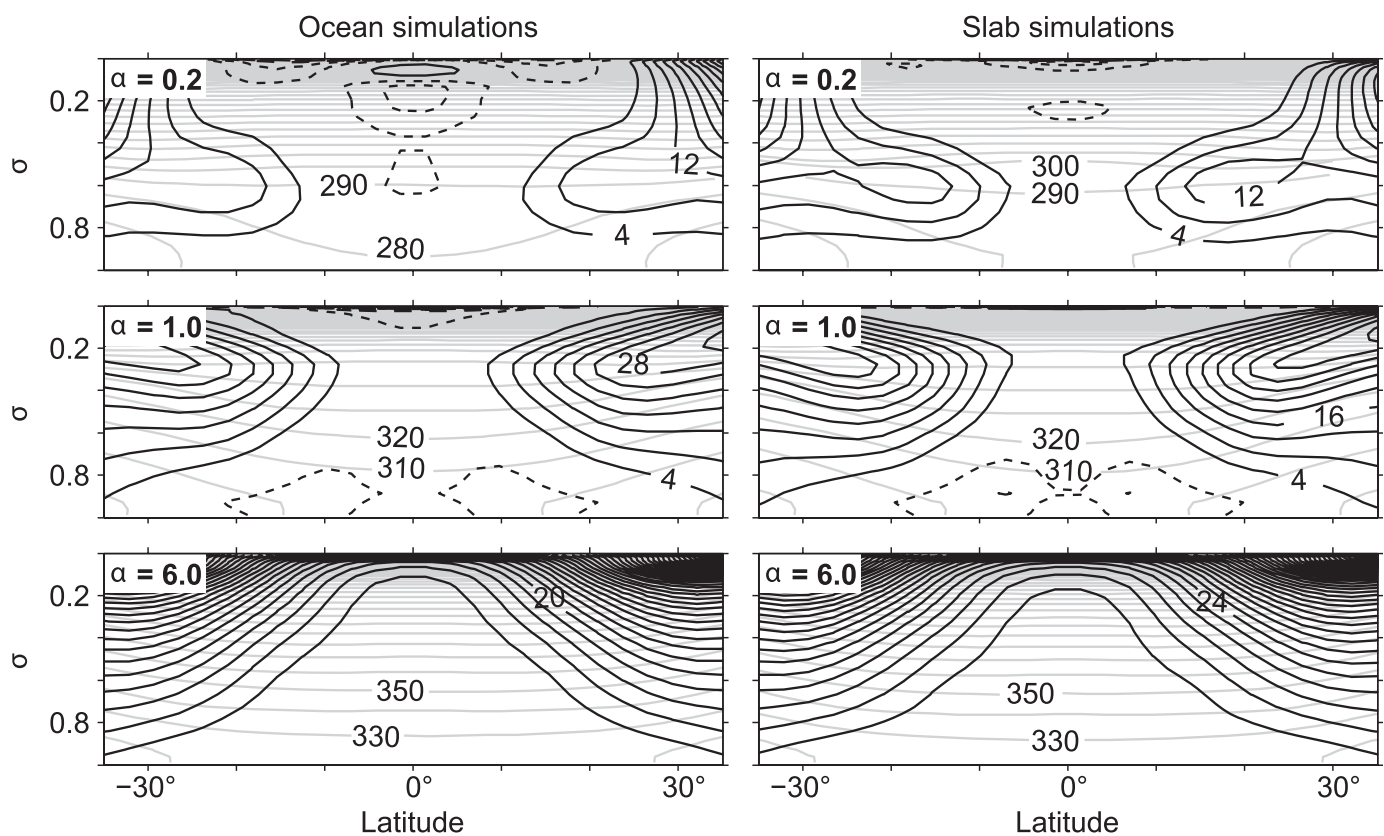

FIG. 3. Mean zonal wind (black contours) and potential temperature (gray contours). Dashed black contours indicate easterlies and solid black contours indicate westerlies. Contour intervals are $4 \mathrm{~m} \mathrm{~s}^{-1}$ for zonal wind and $10 \mathrm{~K}$ for potential temperature. Rows show (top) the coldest $(\alpha=0.2)$, (middle) the reference $(\alpha=1.0)$, and (bottom) the warmest $(\alpha=6.0)$ simulation both (left) with and (right) without ocean heat transport

the mean poleward mass flux and the eddy momentum flux divergence, which are linearly related since Ro is small, differ by less than $25 \%$ between the two reference simulations. This demonstrates that the influence of ocean heat transport on the atmosphere is stronger in the deep tropics than in the subtropics, as expected given that the ocean heat flux is weak in the subtropics.

Ocean heat transport is the only process that we found is able to produce Hadley circulations with a strength and in a dynamical regime similar to Earth's. Otherwise, for instance, the radiative transfer formulation would have to be made unphysical (e.g., longwave optical thickness increasing away from the equator) to reduce the strength of the Hadley circulation to Earthlike values while leaving the eddy momentum flux divergence nearly unchanged. The necessity of including a representation of ocean heat transport is also seen in more comprehensive climate simulations, which otherwise produce unrealistically strong Hadley circulations (e.g., Herweijer et al. 2005).

\section{Variations of climate}

To investigate how the Hadley circulation responds to climate changes, we conducted a series of simulations with and without ocean heat transport in which we varied the prescribed longwave optical thickness. Insolation at the top of the atmosphere and absorption of solar radiation within the atmosphere are kept fixed, so that the only changes in the energy budget come from changes in the longwave radiation (i.e., we do not take into account factors such as changes in the shortwave absorption owing to changes in the atmospheric concentration of water vapor). The longwave optical thickness is varied by rescaling the optical thickness in the reference simulation - a function of latitude and pressureby a constant factor $\alpha$ as in O'Gorman and Schneider (2008b). Reducing the optical thickness $(\alpha<1)$ produces climates colder than the reference climate; increasing it $(\alpha>1)$ produces warmer climates. The rescaling factor $\alpha$ is set to 18 different values ${ }^{1}$ ranging from 0.2 to 6.0 . Each simulation is run for 1000 days. The simulated climates that result from these variations have global-mean surface temperatures between 260 and $315 \mathrm{~K}$. The poleto-equator surface temperature contrast decreases monotonically as the climate warms, in both the slab and ocean simulations, from $\sim 75 \mathrm{~K}$ in the coldest simulation to $\sim 23 \mathrm{~K}$ in the warmest simulation. The surface temperature contrast decreases largely because the poleward latent heat transport by eddies increases (O'Gorman and Schneider 2008b).

\footnotetext{
${ }^{1}$ The values for $\alpha$ are $0.2,0.3,0.4,0.5,0.6,0.7,0.8,0.9,1.0,1.2$ $1.4,1.6,1.8,2.0,2.5,3.0,4.0$, and 6.0.
} 


\section{a. Hadley circulation mass flux}

\section{1) STRENGTH}

In the simulations with ocean heat transport, the latitude of peak poleward mass flux in the upper branch of the Hadley circulation $\varphi_{c}$, or the extremum of the mass flux streamfunction, shifts equatorward as the climate warms, from $11.0^{\circ}$ in the coldest simulation to $8.9^{\circ}$ in the reference simulation and to $6.6^{\circ}$ in the warmest simulation (Fig. 2, left column). In the simulations without ocean heat transport, it is closer to the equator and hardly changes as the climate warms, varying from $5.8^{\circ}$ in the coldest simulation to $6.7^{\circ}$ in the reference simulation to $6.6^{\circ}$ in the warmest simulation (Fig. 2, right column). On the other hand, it varies little in height, being confined between $\sim 500$ and $\sim 700 \mathrm{hPa}$ over the wide range of climates we simulated.

To compare the strength of the Hadley circulation over the entire range of climates we simulated, we compare the absolute values of the mass flux streamfunction evaluated at the (varying) latitude of the streamfunction extremum but at the (fixed) level $\sigma_{c}=0.7\left(\sigma_{c}\right.$ is the pressure at $p_{c}$ normalized by its local value at the surface). Choosing the lowest level of the extremum of the mass flux streamfunction in the simulations for evaluation of the streamfunction reduces noise that would otherwise arise because of the vertical displacement of the center of the Hadley circulation. Figure 4 shows the results. As is already evident in Fig. 2, the Hadley circulation strength changes nonmonotonically with climate in both the ocean and slab simulations. In the ocean simulations, it is largest (102 Sv) in the reference climate $(\alpha=1.0$, global-mean surface temperature $=290 \mathrm{~K}$ ) and is considerably lower in much colder and in much warmer climates. The behavior in the slab simulations is similar, although the maximum $(188 \mathrm{~Sv})$ occurs in a colder climate $(\alpha=0.6$, global-mean surface temperature $=281 \mathrm{~K})$. The maximum is broader in the ocean simulations than in the slab simulations. For instance, the strength of the Hadley circulation varies by less than $15 \%$ of its maximum value for ocean simulations with global-mean surface temperatures between 281 and $304 \mathrm{~K}$. This range of weak Hadley circulation sensitivity is narrower in the slab simulations and is shifted to colder simulations, with temperatures between 273 and $290 \mathrm{~K}$.

The strength of the Hadley circulation is most strongly affected by ocean heat transport in the coldest simulations, with a steady decrease of the dynamical importance of ocean heat transport as the climate warms. For instance, the Hadley circulation in the ocean simulations is weaker than in the slab simulations by $58 \%$ in the coldest simulation and by $37 \%$ in the reference simulation, but only by less than $1 \%$ in the warmest simulation.

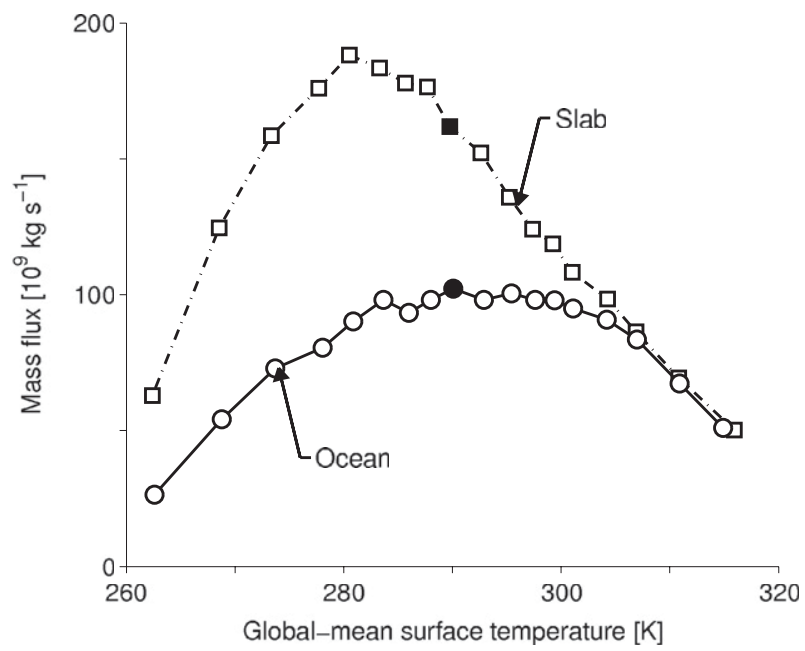

FIG. 4. Strength of the Hadley circulation in simulations with (solid with circles) and without (dashed-dotted with squares) ocean heat transport. Shown is the absolute value of the mass flux streamfunction at the latitude of its extremum and at the level $\sigma_{c}=$ 0.7 , averaged over both hemispheres. Filled symbols identify the reference simulations in this and subsequent figures.

The difference in Hadley circulation strength between the ocean and slab simulations becomes small in the warmest climates because the Hadley circulations there are relatively weak and the meridional surface temperature gradients in the tropics and subtropics are small, so that the ocean heat transport (3) becomes inefficient. For example, in the warmest ocean simulation, the surface temperature contrast between the equator and the subtropical termini of the Hadley circulation amounts to only $4.5 \mathrm{~K}$, compared with $8.0 \mathrm{~K}$ in the reference simulation. We expect the reduced importance of ocean heat transport in warm climates to be a general result, given the robustness of the mechanisms involved.

\section{2) EDDY COMPONENT AND ROSSBY NUMBER}

\section{(i) Theory}

For the analysis of dynamical mechanisms responsible for these changes, it is helpful to decompose the Hadley circulation strength into a component associated with eddy momentum fluxes and a component associated with nonlinear mean momentum fluxes (Schneider and Bordoni 2008). By vertical integration of the zonal momentum balance (1) over the upper branch of the Hadley circulation, from the top of the atmosphere to some pressure $p_{c}$ above the top of the planetary boundary layer, it follows that the mass flux streamfunction $\Psi=$ $\Psi_{e}(1-\langle\mathrm{Ro}\rangle)^{-1}$ near the center of the Hadley cells can be decomposed into a component $\Psi_{e}$ associated with eddy momentum fluxes and a bulk Rossby number $\langle$ Ro $\rangle$ associated with nonlinear mean momentum fluxes, with 


$$
\begin{gathered}
\Psi(\phi, p)=\frac{2 \pi a \cos \varphi}{g} \int_{0}^{p} \bar{v} d p^{\prime}, \\
\Psi_{e}(\phi, p)=\frac{2 \pi a \cos \varphi}{f g} \int_{0}^{p} S_{e} d p^{\prime},
\end{gathered}
$$

where

$$
S_{e}=\frac{1}{a \cos ^{2} \varphi} \partial_{\varphi}\left(\overline{u^{\prime} v^{\prime}} \cos ^{2} \varphi\right)+\partial_{p}\left(\overline{\omega^{\prime} v^{\prime}}\right)
$$

and

$$
\langle\operatorname{Ro}\rangle(\phi, p)=\frac{\Psi-\Psi_{e}}{\Psi} .
$$

The bulk Rossby number is a nondimensional measure of the proximity of the Hadley circulation to the angular momentum-conserving limit, or of the relative importance of nonlinear mean momentum fluxes and eddy momentum fluxes in the zonal momentum balance. As for the local Rossby number, in the angular momentumconserving limit, $\Psi_{e}$ vanishes so that $\langle$ Ro $\rangle \rightarrow 1$. In the eddy-driven limit, eddy momentum fluxes dominate, so that $\Psi \rightarrow \Psi_{e}$ and $\langle$ Ro $\rangle \rightarrow 0$. However, even $\langle$ Ro $\rangle \approx 1$ would not necessarily imply that all eddy fluxes are unimportant for the Hadley circulation, only that eddy momentum fluxes play a negligible role in the zonal momentum balance. Eddy energy fluxes may still influence the Hadley circulation through their effect on thermodynamic balances. We examine the behavior of the eddy component and the bulk Rossby number in both the slab and ocean simulations.

\section{(ii) Bulk Rossby number}

The bulk Rossby number, evaluated at the latitude of the streamfunction extremum $\phi_{c}$ and at the fixed level $\sigma_{c}=0.7$, increases nearly monotonically with globalmean surface temperature: in simulations with (without) ocean heat transport, it increases from $0.18(0.32)$ in the coldest climate to $0.41(0.60)$ in the reference climate and to $0.86(0.88)$ in the warmest climate (Fig. 5). Both slab and ocean simulations show a steady transition in the flow regime from low to large Rossby number as the climate warms, although the slab simulations have larger bulk Rossby numbers than the ocean simulations, especially for cold climates (with $\alpha \lesssim 1.0$ ).

An increase in the bulk Rossby number implies an increase in the meridional shear of the zonal wind. The latter can be related to changes in the mean tropospheric zonal-wind shear and in the height of the tropopause. Our simulations indicate that in the deep tropics and near the center of the Hadley circulation, the bulk Rossby number increases primarily because the tropopause height

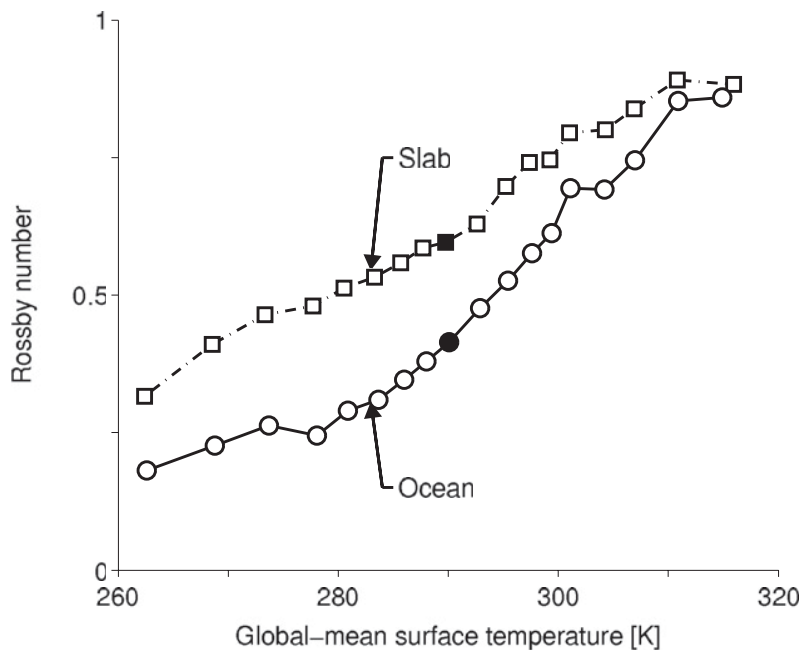

FIG. 5. Bulk Rossby number in the simulations with (solid with circles) and without (dashed-dotted with squares) ocean heat transport. All quantities are evaluated at the latitude of the streamfunction extremum and at the level $\sigma_{c}=0.7$, and are averaged over both hemispheres.

increases. But the mean tropospheric zonal-wind shear in the subtropics also increases as the climate warms, and this likewise contributes to an increase in the bulk Rossby number there. The larger meridional shear of the zonal wind in the warm climates is consistent with a strengthening of the subtropical jet as the climate warms; the jet strengthens in simulations with (without) ocean heat transport from $11(15) \mathrm{m} \mathrm{s}^{-1}$ in the coldest climate and $29(36) \mathrm{m} \mathrm{s}^{-1}$ in the reference climate to $121(125) \mathrm{m} \mathrm{s}^{-1}$ in the warmest climate (Fig. 3) - an indication of the reduced eddy damping of the zonal winds in the warm climates.

\section{(iii) Eddy component}

The eddy component, also evaluated at the latitude of the streamfunction extremum $\phi_{c}$ and at the fixed level $\sigma_{c}=0.7$, changes nonmonotonically as the global-mean surface temperature increases (Fig. 6). It has nearly equal strength in the slab and in the ocean simulations. It is maximal near the same relatively cold climate regardless of ocean heat transport (ocean: $\alpha=0.7$, global-mean surface temperature $\sim 284 \mathrm{~K}$; slab: $\alpha=0.8$, global-mean surface temperature $\sim 287 \mathrm{~K}$ ), with nearly the same maximum values $(\sim 63$ and $65 \mathrm{~Sv})$. The relative importance of the eddy component in driving the Hadley circulation generally decreases as the climate warms, as already indicated by the local Rossby numbers (Fig. 2).

The nonmonotonic behavior of the eddy momentum flux divergence in the tropics, which is maximal near the reference simulation, is directly related to changes in eddy activity in the extratropics. Globally integrated, the eddy momentum flux divergence in tropical and polar 


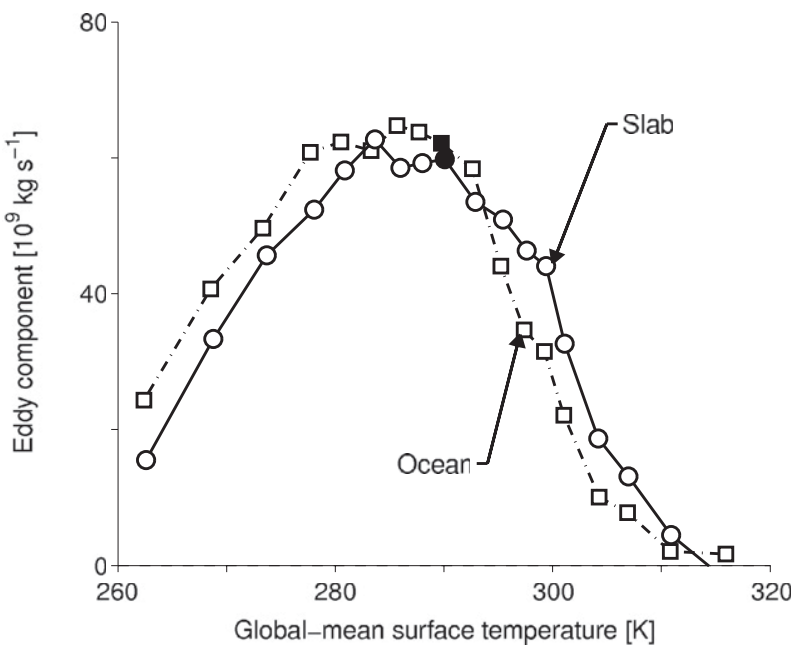

FIG. 6. Eddy component of the mass flux streamfunction in the simulations with (solid with circles) and without (dashed-dotted with squares) ocean heat transport. All quantities are evaluated at the latitude of the streamfunction extremum and at the level $\sigma_{c}=$ 0.7 , and are averaged over both hemispheres.

regions equals the eddy momentum flux convergence in midlatitudes, where baroclinic instability preferentially occurs. Dimensional analysis suggests that the eddy momentum flux convergence scales like the product of the eddy kinetic energy and a characteristic inverse length scale (Schneider and Walker 2008),

$$
\left\langle\partial_{y} \overline{u^{\prime} v^{\prime}}\right\rangle \sim \frac{\left\langle\overline{u^{\prime 2}}+\overline{v^{\prime 2}}\right\rangle}{L_{e}} .
$$

Here, $\langle\cdot\rangle$ is the average over a baroclinic zone. In our simulations, variations in eddy kinetic energy dominate variations in eddy length scales, so that the eddy kinetic energy and eddy momentum flux convergence vary linearly with each other (Fig. 7). In addition, the eddy kinetic energy scales with the mean available potential energy (MAPE), as shown in Fig. 7. The variations of the latter, in turn, can be decomposed into components associated with the meridional temperature gradient $\left\{\left\langle\partial_{y} \bar{T}\right\rangle\right\}$, inverse dry static stability $\{\Gamma\}\left(\Gamma=-\kappa / p\left\langle\partial_{p} \bar{\theta}\right\rangle^{-1}\right)$, thickness of the troposphere $\Delta p_{t}=p_{s}-p_{t}$, and the width of the baroclinic zone or eddy length scale $L_{e}$, as shown by O'Gorman and Schneider (2008a) for the slab simulations. Here, $\{\cdot\}$ is a vertical average over the troposphere in the baroclinic zone, $p_{s}$ is surface pressure, and $p_{t}$ tropopause pressure. Combining these terms gives

$$
\left\langle\partial_{y} \overline{u^{\prime} v^{\prime}}\right\rangle \sim \Delta p_{t}\left\{\left\langle\partial_{y} \bar{T}\right\rangle\right\}^{2}\{\Gamma\} L_{e} .
$$

As shown by O'Gorman and Schneider for a warming climate in the slab simulations, the nonmonotonic behavior of the static stability, the steady increase in the

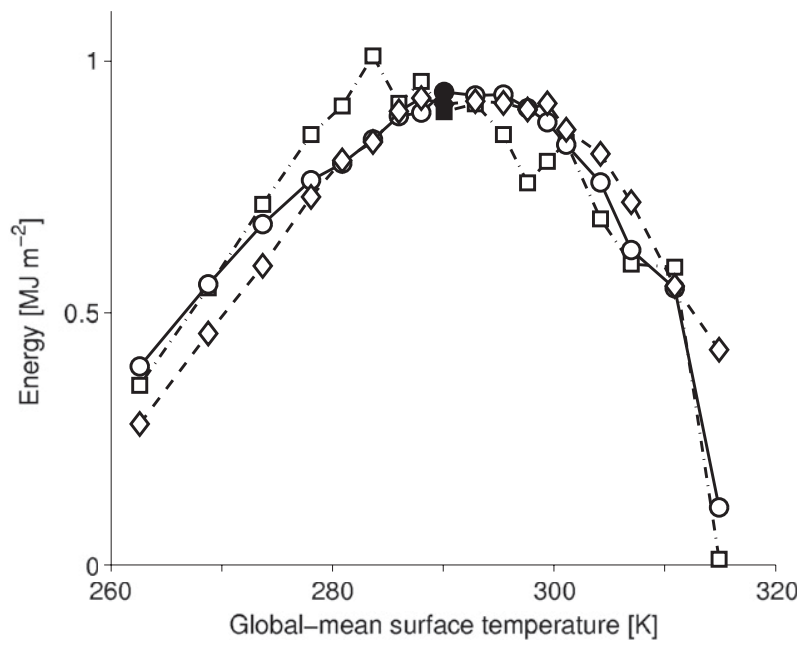

FIG. 7. Mean available potential energy (dashed-dotted with squares) and eddy kinetic energy (solid with circles) integrated over baroclinic zones, and globally integrated positively signed eddy momentum flux divergence (dashed with diamonds). Baroclinic zones are defined as the regions within $15^{\circ}$ of the latitude of the maximum meridional heat flux at the level $\sigma=0.84$. We computed MAPE using the same conventions as in O'Gorman and Schneider (2008a). Quantities are averaged over both hemispheres.

thickness of the troposphere (tropopause height), and the decrease in the meridional temperature gradient all contribute to the nonmonotonic changes in MAPE and thus to the nonmonotonic variations of $\Psi_{e}$ shown in Fig. 6. The same conclusions apply to the ocean simulations, as low-latitude ocean heat transport has only a minimal impact on the extratropical dynamics that control MAPE, and integrals of the eddy angular momentum flux divergence are nearly equal in the slab and ocean simulations. Nevertheless, in the warmest simulations with or without ocean heat transport, the eddy momentum flux divergence in the tropics differs significantly from the eddy momentum flux convergence in midlatitudes. This arises because a superrotating jet appears in the equatorial region, with associated eddy momentum flux convergence at the equator-likely caused by convectively generated Rossby waves, as in Schneider and Liu (2009) —which partially balances the eddy momentum divergence in the subtropics. A similar superrotating jet appears in the more comprehensive simulations of warm climates by Caballero and Huber (2010).

\section{b. Hadley circulation extent}

Several diagnostics have been used to define the subtropical terminus of the Hadley circulation. Reichler (2009) distinguishes two groups. The first comprises dynamical quantities, namely (i) the latitude at which the mass flux streamfunction changes sign, (ii) the latitude at which the mean zonal surface wind changes from easterly to westerly, and (iii) the latitude of the subtropical jets. The second 

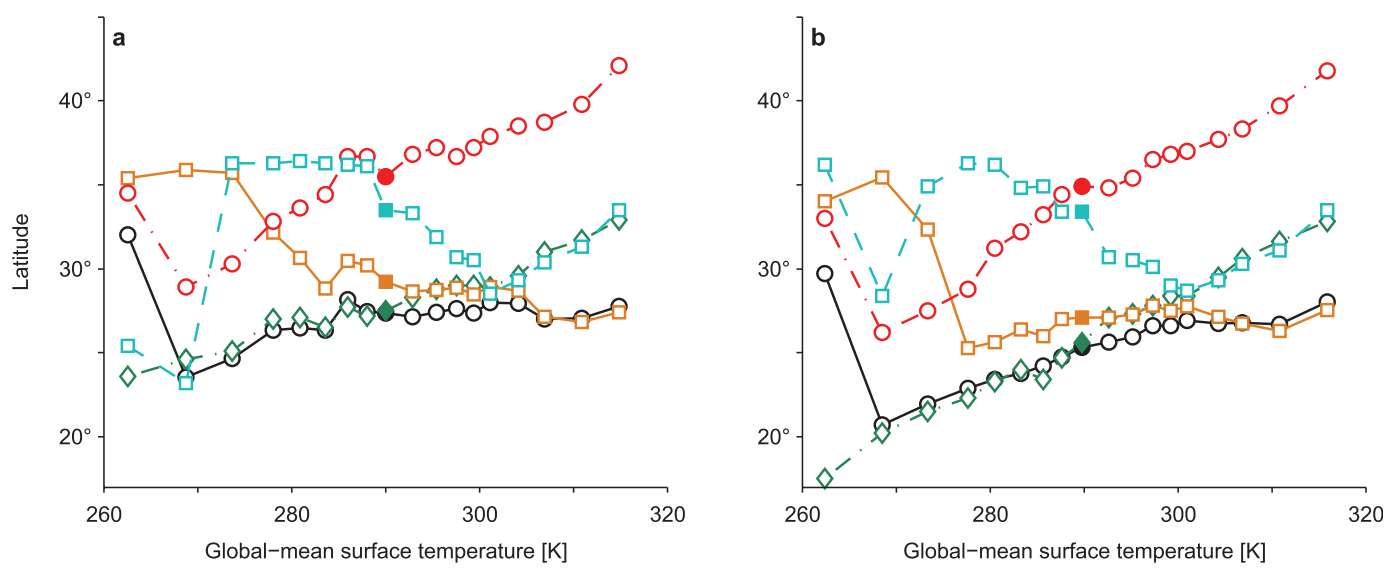

FIG. 8. Extent of the Hadley circulation vs global-mean surface temperature. The extent is defined as the latitude where (i) the mass flux streamfunction at the level $\sigma_{c}=0.7$ changes sign (solid black with circles), (ii) the mean zonal wind at the lowest level of the atmosphere vanishes (solid orange with squares), (iii) the mean zonal wind near the tropopause has a maximum (dashed-dotted green with diamonds), (iv) mean evaporation equals precipitation (dashed-dotted red with circles), and (v) the tropopause rapidly changes height (dashed cyan with squares). Based on quantities averaged over both hemispheres. Simulations (a) with and (b) without ocean heat transport.

comprises derived quantities, namely (iv) the poleward boundary of the subtropical arid zone, where precipitation balances evaporation in the zonal mean ( $\mathrm{Lu}$ et al. 2007), (v) the poleward boundary of the tropical tropopause regime (Seidel and Randel 2007), (vi) the poleward boundary of the tropical ozone regime (Hudson et al. 2006), and (vii) the poleward boundary of the tropical outgoing longwave radiation regime ( $\mathrm{Hu}$ and Fu 2007). On average, these diagnostics suggest that in the past 30 years, the latitude band occupied by the Hadley circulation in the Northern and Southern Hemispheres together has widened at a rate of $\sim 1.4^{\circ}$ decade $^{-1}$ (Reichler 2009). However, these trends exhibit large scatter; depending on the diagnostic and data source used, they vary between $\sim 0.3^{\circ}$ decade $^{-1}$ (Archer and Caldeira 2008) and 3.0 $0^{\circ}$ decade $^{-1}$ (Rosenlof 2002; Seidel and Randel 2007).

Additionally, these diagnostics do not necessarily change in lockstep as the climate changes, even if observational and modeling uncertainties were removed. The diagnostics are not simply related, and they are generally affected by different processes. Here, we examine how the extent of the Hadley circulation changes in our simulations, comparing diagnostics (i) $-(\mathrm{v})$, which are well defined in the idealized GCM. We describe simulations with ocean heat transport (Fig. 8a), but the presence or absence of ocean heat transport does not qualitatively affect the extent of the Hadley circulation or its rate of change with climate (cf. Figs. 8a,b).

(i) The subtropical latitude at which the mass flux streamfunction is zero may be considered the most natural definition of the terminus of the Hadley circulation. This latitude varies with height, especially in the warmest simulations: for instance, Fig. 2 (bottom row) suggests that the poleward terminus is about $5^{\circ}$ farther poleward near the tropopause than near the boundary layer top. Here, we evaluate it at the level $\sigma_{c}=0.7$ (as for other quantities before) because this fixed level is close to the level of the streamfunction extremum for all climates we simulated. The corresponding latitude generally shifts poleward as the climate warms, more rapidly in colder climates than in warmer climates (a prominent exception being the coldest simulation). However, the rate of expansion of the Hadley circulation according to this metric is modest. Averaged over the 11 simulations centered on the reference simulation $(0.5 \leq \alpha \leq 2.0$, global-mean surface temperatures between 281 and $301 \mathrm{~K}$ ), the rate of expansion is only $0.07^{\circ} \mathrm{K}^{-1}$ relative to the global-mean surface temperature increase (Table 1). However, the results change qualitatively if in place of the latitude at which the streamfunction changes sign we use the latitude at which the absolute streamfunction value is below a low threshold value (e.g., $25 \mathrm{~Sv}$ ) or below a fraction of its extremal value (e.g., 20\%). For instance, if we choose a 25-Sv threshold value, shown in Fig. 2 for three climates, the Hadley cells contract in the warmer simulations (with globalmean surface temperature greater than $299 \mathrm{~K}$ ). Comparing changes in the terminus of the Hadley circulation obtained from the mass flux streamfunction therefore depends sensitively on how it is defined. 
TABLE 1. Location of subtropical Hadley circulation terminus in reference simulation and trend (expansion rate) normalized by global-mean surface temperature increase. The expansion rates are averaged over 11 simulations centered on the reference simulations $(0.5 \leq \alpha \leq 2.0)$. The terminus is determined according to different diagnostics: (i) zero of the mass flux streamfunction $\Psi$; (ii) zero of the mean zonal surface wind $u_{s}$; (iii) maximum of the mean zonal wind at the level of the midlatitude tropopause $u_{t}$; (iv) latitude at which zonal-mean precipitation equals evaporation $(\bar{P}=\bar{E})$; and (v) extremum of the meridional gradient of the tropopause height $H_{t}$. The quantities are shown for the ocean and the slab simulations and are averaged over both hemispheres.

\begin{tabular}{cccccc}
\hline \hline & \multicolumn{2}{c}{ Ocean simulations } & & \multicolumn{2}{c}{ Slab simulations } \\
\cline { 2 - 3 } \cline { 5 - 6 } Diagnostic & Lat $\left(^{\circ}\right)$ & Trend $\left({ }^{\circ} \mathrm{K}^{-1}\right)$ & & Lat $\left(^{\circ}\right)$ & Trend $\left({ }^{\circ} \mathrm{K}^{-1}\right)$ \\
\hline$\Psi$ & 27.4 & 0.07 & & 25.3 & 0.17 \\
$u_{s}$ & 29.2 & -0.14 & & 27.1 & 0.11 \\
$u_{t}$ & 27.5 & 0.08 & & 25.6 & 0.26 \\
$\bar{P}=\bar{E}$ & 35.5 & 0.22 & & 34.9 & 0.35 \\
$H_{t}$ & 33.5 & -0.34 & & 33.4 & -0.33 \\
\hline
\end{tabular}

(ii) The latitude at which the mean zonal surface wind changes from easterlies in the tropics to westerlies in the extratropics shifts equatorward by about $6^{\circ}$ between the coldest and the reference simulation; it remains near the zero of the mass flux streamfunction for climates warmer than the reference simulation. Near the reference simulation, the rate of expansion is $-0.14^{\circ} \mathrm{K}^{-1}$ relative to the globalmean surface temperature increase (Table 1).

(iii) The latitude of the subtropical jet, defined as the latitude at which the mean zonal wind at the level of the midlatitude tropopause is maximal, shifts poleward in a similar way as the zero of the mass flux streamfunction in simulations colder than the reference simulation; however, it shifts poleward more rapidly in warmer simulations. For all simulations except the coldest, the subtropical jet remains within $5^{\circ}$ of the zero of the mass flux streamfunction. Near the reference simulation, the rate of expansion is $0.08^{\circ} \mathrm{K}^{-1}$ relative to the globalmean surface temperature increase (Table 1 ).

(iv) The latitude at which the zonal-mean precipitation equals evaporation, $\bar{P}=\bar{E}$, is generally located $\geq 8^{\circ}$ poleward of the zero of the mass flux streamfunction. It shifts poleward from $29^{\circ}$ to $42^{\circ}$ as the climate warms, implying a rate of expansion about 3 times as large as that obtained from the zero of the mass flux streamfunction (Table 1).

(v) The poleward boundary of the tropical tropopause regime is determined as the closest latitude to the equator at which the meridional gradient of the tropopause height has a local extremum. It shifts equatorward as the climate warms for simulations with global-mean surface temperature between 273 and $301 \mathrm{~K}$, from $36^{\circ}$ to $28^{\circ}$. This contraction is greater for simulations with global-mean surface temperatures between 288 and $301 \mathrm{~K}$ (Fig. 8a). However, it shifts poleward for warmer climates, in which it closely follows the location of the subtropical jet. Near the reference simulation, the rate of expansion is $-0.34^{\circ} \mathrm{K}^{-1}$ relative to the globalmean surface temperature increase (Table 1).

Hence, there are large differences in the subtropical terminus of the Hadley circulation and its shifts with climate according to the five diagnostics. Even the signs of the expansion rate can differ from diagnostic to diagnostic. Furthermore, the rate of expansion of the Hadley circulation according to the zero of the mass flux streamfunction-arguably the dynamically most relevant diagnostic - is considerably smaller than the rate of expansion according to the other diagnostics. Results from the slab simulations are similar (see Fig. 8b for comparison), albeit with generally narrower Hadley circulations that expand poleward more rapidly as the climate warms (Table 1). The zero of the mass flux streamfunction is the diagnostic that is the most sensitive to the presence of ocean heat transport, as shown on Table 1, leading to expansion rates that differ by about a factor of 3 between ocean and slab simulations near the reference simulations. The rates of expansion of the Hadley circulation according to the diagnostics shown in Table 1 differ because they are controlled by different mechanisms. For example, the latitude at which zonal-mean precipitation equals evaporation depends strongly on the eddy fluxes of water vapor out of low latitudes into middle latitudes. However, these fluxes do not bear any simple relation to the zero of the mass flux streamfunction. In simulations with global-mean surface temperatures $\$ 288 \mathrm{~K}$, the zero of the mean zonal surface wind lies $2^{\circ}-12^{\circ}$ farther poleward of the zero of the mass flux streamfunction (Figs. 8a,b). In these colder simulations, the eddy momentum flux divergence at upper levels in the shallow troposphere and in the stratosphere extends poleward of the subtropical jet, between it and a midlatitude jet located $10^{\circ}-30^{\circ}$ poleward (see, e.g., Fig. 2, top row). Because the vertically integrated eddy momentum flux divergence at these latitudes (where the local Rossby number is small) balances the drag on the mean zonal surface wind, surface easterlies extend poleward of the zero of the mass flux streamfunction in the troposphere. As the climate warms, the eddy momentum flux divergence in the midlatitude stratosphere weakens, and the zero of the mean zonal surface wind moves closer to the zero of the mass flux streamfunction. 

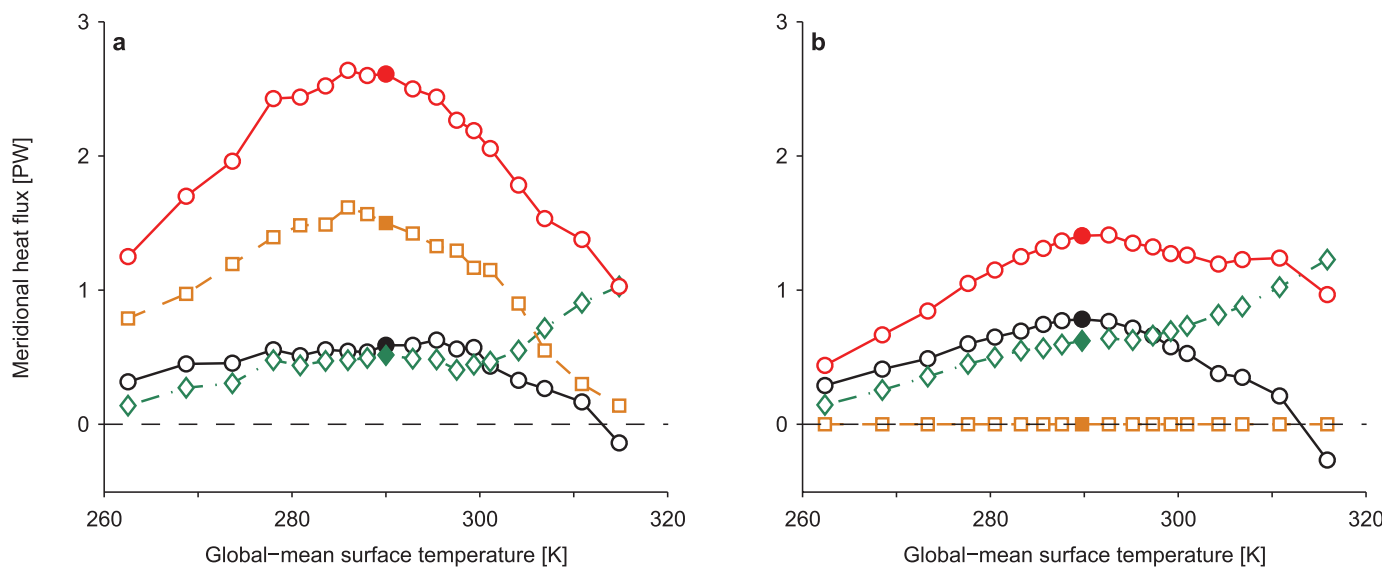

FIG. 9. Vertically integrated heat fluxes evaluated at the center of the Hadley cells (latitude of the streamfunction extremum). Total ocean-atmosphere heat flux (red solid with circles) and its components: mean component (black solid with circles) and eddy component (green dashed-dotted with diamonds) of the moist static energy flux; ocean heat flux (orange dashed with squares). The fluxes are averaged over both hemispheres. Simulations (a) with and (b) without ocean heat transport.

Simulations of global warming scenarios with comprehensive GCMs suggest that the zero of the mass flux streamfunction shifts poleward at rates of about $0.5^{\circ}$ per 1-K global-mean surface temperature increase ( $\mathrm{Lu}$ et al. 2007). Idealized GCMs forced by varying sea surface temperatures (SSTs) suggest that the zero of the mass flux streamfunction shifts poleward at rates of about $0.2^{\circ}-0.25^{\circ}$ per $1-\mathrm{K}$ global-mean surface temperature increase (Frierson et al. 2007). This is similar to the expansion rate $\left(0.17^{\circ} \mathrm{K}^{-1}\right)$ in our slab simulations near the reference climate (Table 1 ) and up to $\sim 3$ times greater than the expansion rate $\left(0.07^{\circ} \mathrm{K}^{-1}\right)$ in our ocean simulations. The discrepancies between the rate of expansion of the Hadley circulation in comprehensive and idealized GCMs may be due to the presence of continents as well as of a seasonal cycle.

\section{c. Energy transport}

The strength of the Hadley circulation may also be measured in terms of how much energy it transports meridionally. If one neglects the small transport of kinetic energy, the total energy transport in the atmosphere is equal to the transport of moist static energy $s=s_{d}+L q$, which consists of contributions from the dry static energy $s_{d}=c_{p} T+g z$ and from the latent heat $L q$ (symbols have their conventional meaning; see, e.g., Peixoto and Oort 1992, chapter 13). The Hadley circulation transports dry static energy poleward (because the dry static energy in the upper branch is higher than that in the lower branch), and it transports latent heat equatorward (because the specific humidity in the lower branch is greater than that in the upper branch). The net transport of moist static energy is the residual of these partially compensating fluxes, and it is generally much smaller than the fluxes individually.

The moist static energy flux associated with the Hadley circulation (its mean component) in the ocean simulations is shown in Fig. 9a, along with the eddy flux of moist static energy (its eddy component) and the ocean heat flux, all evaluated at the center of the Hadley cells. The eddy component of the moist static energy flux is comparable with the mean component over a wide range of climates, although the eddy components of the dry static energy and latent heat fluxes individually are considerably smaller than the mean components: while the mean components of the dry static energy and latent heat fluxes partially compensate, the eddy fluxes of both dry static energy and latent heat are directed poleward (downgradient). Both the mean and the eddy components of the moist static energy flux strengthen as the climate warms for simulations with global-mean surface temperatures below $300 \mathrm{~K}$. For simulations with globalmean surface temperature above $300 \mathrm{~K}$, the mean component weakens sharply and even becomes negative in the warmest simulations, while the eddy component continues to increase essentially monotonically. The sum of the ocean heat flux and the atmospheric moist static energy flux at the center of the Hadley cells, the total atmosphere-ocean heat flux, peaks near the reference simulation (Fig. 9a). Over the wide range of climate we simulated, the partition between the mean component of the moist static energy flux and the ocean heat transport remains nearly unchanged; its value, which varies between $\sim 25 \%$ and $\sim 40 \%$, is in good agreement with the prediction of Held (2000). The changes with climate of the total heat flux resemble those of the mass 
flux streamfunction (cf. Figs. 4 and 9a), primarily because the ocean heat flux dominates the total heat flux, and its strength is tightly coupled to the strength of the Hadley circulation.

The moist static energy flux in the slab simulations behaves similarly to that in the ocean simulations (cf. Figs. 9a,b). However, both the mean and eddy components are up to $\sim 60 \%$ larger in the slab simulations than in the ocean simulations. But even the larger moist static energy fluxes in the slab simulations do not compensate for the lack of ocean heat transport. For instance, at the reference simulations, the total heat transport is $40 \%$ smaller in the slab simulations. This contrasts with the heat fluxes at the subtropical termini of the Hadley circulation, which are less sensitive to ocean heat transport. For instance, the difference between the moist static energy fluxes in the slab and ocean reference simulations at the center of the Hadley circulation is $0.45 \mathrm{PW}$, which is significantly larger than the corresponding difference at the termini of the Hadley circulation (only 0.10 PW). It implies that most of the additional heat carried by the atmosphere-ocean system in the ocean simulations is radiated to space in the subtropics. ${ }^{2}$

Overall, it is clear that also if measured in terms of how much energy it transports meridionally, the strength of the Hadley circulation can change nonmonotonically with global mean surface temperature. The fact that the energy transport by the Hadley circulation (though not by the total circulation including eddies) can become negative (equatorward) illustrates some of the difficulties that can arise in devising theories for how the mass flux in the Hadley circulation relates to the energy flux.

\section{Conclusions}

We have introduced an idealized GCM with a onedimensional representation of ocean heat transport that is dynamically coupled to the surface wind stress. With this idealized GCM, we have simulated a wide range of climates by varying the optical thickness of a longwave absorber, comparing the simulations with ocean heat transport with otherwise identical previous simulations without ocean heat transport (O'Gorman and Schneider 2008b). Because the ocean heat transport dominates the total ocean-atmosphere heat transport in low latitudes,

\footnotetext{
${ }^{2}$ These results contradict the hypothesis of Trenberth and Stepaniak (2003), according to which extratropical eddy heat fluxes were posited as a driver of the Hadley circulation. In our simulations, changes in the strength of the Hadley circulation between ocean simulations and slab simulations are not accompanied by changes in extratropical eddy heat fluxes that are nearly as large, and the total heat flux is not necessarily "seamless" in the subtropics.
}

it affects the dynamics of the tropical atmosphere. It is necessary to represent ocean heat transport in idealized GCMs to obtain Hadley circulations in a dynamical regime similar to Earth's, with relatively low Rossby numbers at the center of the equinox or annualmean Hadley circulation. Our principal results are as follows:

(i) Ocean heat transport strongly influences the Hadley circulation in climates resembling present-day Earth's and colder. Its influence diminishes as the climate warms and meridional temperature gradients in low latitudes are reduced.

(ii) As the climate warms, the Hadley circulation strengthens in colder simulations but weakens in warmer simulations. In the coldest/warmest simulations, it is $\sim 70 \%$ weaker than in the reference simulations with or without ocean heat transport.

(iii) As the climate warms, the bulk Rossby number at the center of the Hadley circulation increases from 0.2 in the coldest ocean simulations and 0.3 in the coldest slab simulations to 0.9 in the warmest simulations: The coldest simulations are strongly influenced by eddy momentum fluxes but the warmest simulations are closer to angular momentum conserving. Eddy momentum fluxes influence the Hadley circulation more strongly in simulations with ocean heat transport than in simulations without ocean heat transport.

(iv) In simulations with climates resembling presentday Earth's and colder, the Hadley circulation strength scales with the low-latitude divergence of eddy momentum fluxes. The latter in turn scales with the midlatitude mean available potential energy. Thus, midlatitude conditions influence the strength of the tropical circulation.

(v) The Hadley circulation widens by $\sim 5^{\circ}$ latitude between the coldest and warmest simulations. The widening rate is greater in climates resembling present-day Earth's and colder. However, different diagnostics for the extent of the Hadley circulation change with climate in different ways because they are often not dynamically related.

Some of our results-such as the behavior of the Hadley circulation strength-may be modified if additional processes (e.g., a seasonal insolation) and feedbacks (e.g., ice-albedo feedback) are taken into account. Nevertheless, the nonmonotonicity of numerous key variables already in our relatively simple setting and the intermediate values of the bulk Rossby number suggest that an accurate prediction for the changes of the largescale tropical dynamics with climate requires understanding both the changes in the Rossby number and in 
eddy fluxes. Devising a theory that takes both effects into account remains as a challenge.

Acknowledgments. We thank Paul O'Gorman for performing the simulations without ocean heat transport and for helpful comments and discussions. We are grateful for support by the National Science Foundation (Grants ATM-0450059 and AGS-1019211), the Davidow Discovery Fund, and a David and Lucile Packard Fellowship. The simulations were performed on the Division of Geological and Planetary Sciences's Dell cluster at the California Institute of Technology. The program code for the simulations described in this paper, and the simulation results themselves, are available from the authors upon request.

\section{REFERENCES}

Archer, C. L., and K. Caldeira, 2008: Historical trends in the jet streams. Geophys. Res. Lett., 35, L08803, doi:10.1029/ 2008 GL033614.

Bordoni, S., and T. Schneider, 2008: Monsoons as eddy-mediated regime transitions of the tropical overturning circulation. Nat. Geosci., 1, 515-519.

Bourke, W., 1974: A multi-level spectral model. I. Formulation and hemispheric integrations. Mon. Wea. Rev., 102, 687-701.

Caballero, R., 2007: Role of eddies in the interannual variability of Hadley cell strength. Geophys. Res. Lett., 34, L22705, doi:10.1029/2007GL030971.

__ 2008: Hadley cell bias in climate models linked to extratropical eddy stress. Geophys. Res. Lett., 35, L18709, doi:10.1029/2008GL035084.

, and M. Huber, 2010: Spontaneous transition to superrotation in warm climates simulated by CAM3. Geophys. Res. Lett., 37, L11701, doi:10.1029/2010GL043468.

Chen, J., B. E. Carlson, and A. D. Del Genio, 2002: Evidence for strengthening of the tropical general circulation in the 1990s. Science, 295, 838-841.

Dickinson, R. E., 1971: Analytic model for zonal winds in the tropics. II. Variation of the tropospheric mean structure with season and differences between hemispheres. Mon. Wea. Rev., 99, 511-523.

Dima, I. M., and J. M. Wallace, 2003: On the seasonality of the Hadley cell. J. Atmos. Sci., 60, 1522-1527.

Frierson, D. M. W., 2007: The dynamics of idealized convection schemes and their effect on the zonally averaged tropical circulation. J. Atmos. Sci., 64, 1959-1976.

—, I. M. Held, and P. Zurita-Gotor, 2006: A gray-radiation aquaplanet moist GCM. Part I: Static stability and eddy scale. J. Atmos. Sci., 63, 2548-2566.

— J. Lu, and G. Chen, 2007: The width of the Hadley cell in simple and comprehensive general circulation models. Geophys. Res. Lett., 34, L18804, doi:10.1029/2007GL031115.

Haynes, P. H., M. E. McIntyre, T. G. Shepherd, C. J. Marks, and K. P. Shine, 1991: On the "downward control" of extratropical diabatic circulations by eddy-induced mean zonal forces. J. Atmos. Sci., 48, 651-678.

Held, I. M., 2000: The partitioning of the poleward energy transport between the tropical ocean and atmosphere. J. Atmos. Sci., 58, 943-948.
__ and A. Y. Hou, 1980: Nonlinear axially symmetric circulations in a nearly inviscid atmosphere. J. Atmos. Sci., 37, 515-533.

Herweijer, C., R. Seager, M. Winton, and A. Clement, 2005: Why ocean heat transport warms the global mean climate. Tellus, 57A, 662-675.

Hu, Y., and Q. Fu, 2007: Observed poleward expansion of the Hadley circulation since 1979. Atmos. Chem. Phys., 7, 5229-5236.

Hudson, R. D., M. F. Andrade, M. B. Follette, and A. D. Frolov, 2006: The total ozone field separated into meteorological regimes. Part II: Northern Hemisphere mid-latitude total ozone trends. Atmos. Chem. Phys., 6, 5183-5191.

Johanson, C., and Q. Fu, 2009: Hadley cell widening: Model simulations versus observations. J. Climate, 22, 2713-2725.

Kallberg, P., A. Simmons, S. Uppala, and M. Fuentes, 2004: The ERA-40 archive. ERA-40 Project Rep. 17, ECMWF, 31 pp.

Klinger, B. A., and J. Marotzke, 2000: Meridional heat transport by the subtropical cell. J. Phys. Oceanogr., 30, 696-705.

Lee, M. I., M. J. Suarez, I. S. Kang, I. M. Held, and D. Kim, 2008: A moist benchmark calculation for atmospheric general circulation models. J. Climate, 21, 4934-4954.

Lindzen, R. S., and A. V. Hou, 1988: Hadley circulations for zonally averaged heating centered off the equator. J. Atmos. Sci., $\mathbf{4 5}$, 2416-2427.

Lu, J., G. A. Vecchi, and T. Reichler, 2007: Expansion of the Hadley cell under global warming. Geophys. Res. Lett., 34, L06805, doi:10.1029/2006GL028443.

Lu, P., J. P. McCreary Jr., and B. A. Klinger, 1998: Meridional circulation cells and the source waters of the Pacific Equatorial Undercurrent. J. Phys. Oceanogr., 28, 62-84.

McCreary, J. P., Jr., and P. Lu, 1994: Interaction between the subtropical and equatorial ocean circulations: The subtropical cell. J. Phys. Oceanogr., 24, 466-497.

Mitas, C. M., and A. Clement, 2005: Has the Hadley cell been strengthening in recent decades? Geophys. Res. Lett., 32, L03809, doi:10.1029/2004GL021765.

O'Gorman, P. A., and T. Schneider, 2008a: Energy of midlatitude transient eddies in idealized simulations of changed climates. J. Climate, 21, 5797-5806.

— and $\_$, 2008b: The hydrological cycle over a wide range of climates simulated with an idealized GCM. J. Climate, 21, 3815-3832.

— and — 2008c: Weather-layer dynamics of baroclinic eddies and multiple jets in an idealized general circulation model J. Atmos. Sci., 65, 524-535.

Peixoto, J. P., and A. H. Oort, 1992: Physics of Climate. American Institute of Physics, $520 \mathrm{pp}$.

Quan, X.-W., H. F. Diaz, and M. P. Hoerling, 2004: Change in the tropical Hadley cell since 1950. The Hadley Circulation: Past, Present, and Future, H. F. Diaz and R. S. Bradley, Eds., Springer, 85-120.

Reichler, T., 2009: Changes in the atmospheric circulation as indicator of climate change. Climate Change: Observed Impacts on Planet Earth, T. M. Letcher, Ed., Elsevier, 145-164.

Rosenlof, K. H., 2002: Transport changes inferred from HALOE water and methane measurements. J. Meteor. Soc. Japan, $\mathbf{8 0}$, 831-848.

Santer, B. D., and Coauthors, 2005: Amplification of surface temperature trends and variability in the tropical atmosphere. Science, 309, 1551-1556.

Schneider, E. K., 1977: Axially symmetric steady-state models of the basic state for instability and climate studies. Part II. Nonlinear calculations. J. Atmos. Sci., 34, 280-296. 
Schneider, T., 2006: The general circulation of the atmosphere. Annu. Rev. Earth Planet. Sci., 34, 655-688.

_ , and S. Bordoni, 2008: Eddy-mediated regime transitions in the seasonal cycle of a Hadley circulation and implications for monsoon dynamics. J. Atmos. Sci., 65, 915-934.

——, and C. C. Walker, 2008: Scaling laws and regime transitions of macroturbulence in dry atmospheres. J. Atmos. Sci., 65, 2153-2173.

—_, and J. Liu, 2009: Formation of jets and equatorial superrotation on Jupiter. J. Atmos. Sci., 66, 579-601.

Seager, R., and Coauthors, 2007: Model projections of an imminent transition to a more arid climate in southwestern North America. Science, 316, 1181-1184.

Seidel, D. J., and R. J. Randel, 2007: Recent widening of the tropical belt: Evidence from tropopause observations. J. Geophys. Res., 112, D20113, doi:10.1029/2007JD008861.

_ Q. Q. Fu, W. J. Randel, and T. J. Reichler, 2008: Widening of the tropical belt in a changing climate. Nat. Geosci., 1, 21-24.

Sherwood, S. C., 2007: Simultaneous detection of climate change and observing biases in a network with incomplete sampling. J. Climate, 20, 4047-4062.
Simmons, A. J., and D. M. Burridge, 1981: An energy and angularmomentum conserving vertical finite-difference scheme and hybrid vertical coordinates. Mon. Wea. Rev., 109, 758-766.

Tanaka, H. L., N. Ishizaki, and D. Nohara, 2005: Intercomparison of the intensities and trends of Hadley, Walker and monsoon circulations in the global warming projections. SOLA, 1, 77-80.

Trenberth, K. E., and A. Solomon, 1994: The global heat balance: Heat transports in the atmosphere and ocean. Climate Dyn., 10, 107-134.

- and J. M. Caron, 2001: Estimates of meridional atmosphere and ocean heat transports. J. Climate, 14, 3433-3443.

— , and D. P. Stepaniak, 2003: Seamless poleward atmospheric energy transports and implications for the Hadley circulation. J. Climate, 16, 3706-3722.

Uppala, S. M., and Coauthors, 2005: The ERA-40 Re-Analysis. Quart. J. Roy. Meteor. Soc., 131, 2961-3012.

Walker, C. C., and T. Schneider, 2005: Response of idealized Hadley circulations to seasonally varying heating. Geophys. Res. Lett., 32, L06813, doi:10.1029/2004GL022304.

, and — 2006: Eddy influences on Hadley circulations: Simulations with an idealized GCM. J. Atmos. Sci., 63, 3333-3350. 